\title{
$\bigcap$ Marine microbial diversity
}

K. S. Sobhana

Marine Biodiversity Division, Central Marine Fisheries Research Institute, Kochi-682 018

Microbes were the only form of life for the first 2-3 billion years of planetary and biological evolution. Life most likely began in the oceans and marine microorganisms are the closest living descendants of the original forms of life. Early marine microorganisms also helped create the conditions under which subsequent life developed. More than two billion years ago, the generation of oxygen by photosynthetic marine microorganisms helped shape the chemical environment in which plants, animals, and all other life forms have evolved. Macroscopic life and planetary habitability completely depend upon the transformations mediated by complex microbial communities. These microscopic factories both aerobic and anaerobic are the essential catalysts for all of the chemical reactions within the biogeochemical cycles. Their unique metabolisms allow marine microbes to carry out many steps of the biogeochemical cycles that other organisms are unable to complete. The smooth functioning of these cycles is necessary for life to continue on earth.

Microbial life in the sea is extremely diverse, including members of all three domains of life (archaea, bacteria and eukarya) as well as viruses (most authorities do not consider viruses as living organisms). The members of these groups or taxa are distinct in terms of their morphology, physiology and phylogeny and fall into both prokaryotic and eukaryotic domains. Considering the adaptability of microorganisms to grow and survive under varied physico-chemical conditions and their contribution in maintaining the balance in ecosystems, it is pertinent to catalogue their diversity as it exists. The inability to visualize them with the naked eye precludes effective classification.

The diversity of microbial communities varies within habitats as much as between habitats. Marine microbial habitats can be classified based on i) presence of other organisms (Symbiotic, Free living and Biofilm); ii) proximity to ocean surface or sediments (Euphotic: 0-150 m; Mesopelagic: 150 $-1000 \mathrm{~m}$; Bathopelagic : >1000m; Benthos : sediments) and also based on iii) concentration of nutrients and required growth substances (Oligotrophic, Mesotrophic, Eutrophic). However, interfaces tend to be hotspots of diversity and biological activity. Marine microbial habitats at interfaces include the air-water, water-sediment, water-ice, and host macroorganism-water interfaces. The sub-millimeter scale of physical and chemical variability in these habitats poses a serious challenge to studying interface habitats in detail. The fact that variations can even occur within a few millimetres, suggests that microbial diversity encompasses more than the documented evidence available. Hence, biogeography is gaining importance as a field of study from microbial diversity point of interest. Due to the innately small size of the microorganisms, environmental complexity plays a major role in determining diversity. Spatial heterogeneity is likely to lead to the formation of many niches within a habitat. Recent tools like metagenomics aid in biogeography studies by providing information on nucleic acid sequence data, thereby directly identifying microorganisms. Therefore the phylogenetic information can be used to compare microbial diversity profile across habitats. Generally, diversity within a particular location and in a community is called alpha diversity. Beta diversity measures the community composition between two or more locations while gamma diversity applies to a region, across continents and biomes and is larger in size than that used for measuring alpha diversity.

Although microbial diversity is one of the difficult areas of marine biodiversity research, estimation of microbial diversity is required for understanding the biogeography, community assembly and ecological processes. The number of species has been a traditional measure of biodiversity in ecology and conservation, but the biodiversity of an area is much more than the 'species richness'. Diversity prediction can be made using statistical approaches that estimate species number from relatively small sample sizes. Hughes et al. (2001) noted that both rarefaction and 
richness estimators which have been applied to microbial datasets, highlighted the utility of nonparametric estimators in predicting and comparing bacterial species number. Rarefaction and richness estimators rely on a species or operational taxonomic unit (OTU) definition. The limitations of this method are that OTUs are counted equivalently despite the fact that some may be highly divergent and phylogenetically unique, whereas others may be closely related and phylogenetically redundant. Recently, statistical analyses borrowed from population genetics and systematics have been employed and reviewed for use with microbial datasets to estimate species richness and phylogenetic diversity which do not rely on estimation of the frequency of different sequences (Martin, 2001). Reciprocal of Simpson's index (1/D), F-statistics (FST) and phylogenetic grouping of taxa ( $P$ tests) may be used as a measure of diversity, which has been widely used for ecological studies (Stach et al., 2003). These combined uses of species richness and diversity estimates provide information that enables deeper understanding of marine microbial diversity.

During the past two decades, molecular studies using the gene sequences that encode the small subunit rRNA (SSU rDNA) have revealed a wealth of new marine microorganisms that belong to the three domains of life viz., bacteria, archaea and the eukarya. The feeling among marine microbiologists is that of living through an age of discovery with no end in sight. However, most of this new diversity has not yet been described because pure cultures of the organisms behind the sequences are necessary to define a species. Recent studies of microbial diversity have produced spectacular discoveries of previously unknown microorganisms, many of which have major impacts on oceanic processes. Very large populations of picoplankton including diatoms, dinoflagellates, picoflagellates and cyanobacteria are the primary catalysts in carbon fixation, which orchestrate the cycling of nitrogen and form the base of the traditional marine food web. Heterotrophic SAR11 represents the dominant clade in communities of oceansurface bacterioplankton while nonphotosynthetic protists of unknown diversity control the size of picoplankton populations and regulate the supply of nutrients into the ocean's food webs. Communities of Bacteria, Archaea, and Protists account for greater than $90 \%$ of oceanic biomass and $98 \%$ of primary production. Archaea includes unusual microorganisms which grow under extreme environments and differs from bacteria due to lack of peptidoglycan. Both these domains collectively play a significant role in the marine environment.

Modern technologies (molecular techniques and automated fluorescence cell sorting) have demonstrated the great abundance and diversity of microbial life forms in the oceans, and DNA sequencing of environmental genomes (metagenomics) provides evidence of hitherto unrecognized physiological categories among the planktonic microbes.
Since $99 \%$ of the microbial population is considered to be uncultivable, metagenomics assumes importance. Molecular techniques have identified SAR11 as a dominant clade in communities of ocean-surface bacterioplankton. Bacteria in the SAR11 clade (Pelagibacteraceae) make up roughly one in three cells at the ocean's surface. Overall, SAR11 bacteria are estimated to make up between a quarter and a half of all prokaryotic cells in the ocean. SAR11 bacteria are classified as alphaproteobacteria, and include the highly abundant marine species Candidatus Pelagibacter ubique.

Sequence information (eg. 16S rRNA sequences, genome sequences, metagenomes) as well as rRNA targeted probes (eg. Fluorescent In Situ Hybridization - FISH, which allows a visual inspection of phylogenetic groups of cells in a natural sample) have helped to discover many new groups of bacteria. Marine microbial genomics ranging from the study of the genomes of model organisms to the wealth of meta-omics approaches (e.g. metagenomics, metatranscriptomics and metaproteomics) has proven to be very successful to target the second basic ecological question on the role of microbes in the marine environment. Advanced technologies, developed in the recent past, promise to revolutionise the way that we characterize, identify, and study microbial communities. The most advanced tools that microbial ecologists can use for the study of microbial communities include innovative microbial ecological DNA microarrays such as PhyloChip and GeoChip that have been developed for investigating the composition and function of microbial communities. Single Cell Genomics approach, which can be used for obtaining genomes from uncultured phyla, enables the amplification and sequencing of DNA from single cells obtained directly from environmental samples and is promising to revolutionise microbiology.

\section{Microbial taxonomy}

Currently, a polyphasic approach is used to define a microbial species using phenotypic and genotypic properties. Whenever a new taxon is proposed, it is essential that the organism be isolated in pure culture and its characteristic features be tested under standard conditions. All the strains within a species must show similar phenotypes. A designated type strain of a species constitutes the reference specimen for that species. If the $16 \mathrm{~S}$ rDNA sequences of organisms are $\leq 98.5 \%$ identical, they are members of different species. Uncultured microbes cannot be assigned to a definite species since their phenotype is not known; however, they can be assigned a 'Candidatus' designation provided their 16S rRNA sequence subscribes to the principles of identity with known species. A concept applying to a taxon lower than that of the strain is the ecotype - those microorganisms that occupy an ecological niche and are adapted to the conditions of that niche

\section{Numerical Taxonomy}

In numerical taxonomy many (50 to 200) biochemical, morphological, and cultural characteristics, as well as susceptibilities to antibiotics and inorganic compounds, are 
used to determine the degree of similarity between organisms. The coefficient of similarity or percentage of similarity between strains (where strain indicates a single isolate from a specimen) is then calculated. A dendrogram or a similarity matrix is constructed that joins individual strains into groups and places one group with other groups on the basis of their percentage of similarity. While $16 \mathrm{~S}$ rDNA sequences have attracted attention in recent times as sole means of bringing out the uniqueness of a species; numerical taxonomy (based on phenotypic traits of a large number of species) compares favourably with that of genotypic data and, indeed, is in alignment with the modern taxonomy

\section{Polyphasic taxonomy}

In polyphasic approach of microbial taxonomy, phenotypic, genotypic and phylogenetic information are described as accurately as possible. The phenotypic information comes from the colony characteristics, cell type, cell wall-type, pigmentation patterns, proteins and other chemotaxonomic markers while genotypic features are derived from the nucleic acids (DNA/RNA). Phylogenetic information is obtained from studying sequence similarities of the $16 \mathrm{~S}$ rRNA or $23 \mathrm{~S}$ rRNA genes in case of bacteria and 18S rRNA in case of fungi. Many types of molecules are used for delineating and describing a taxon; some are mandatory (16S rRNA genes, phenotypes, chemotaxonomy) while others are optional (amino-acid sequencing of certain protein products, DNA-DNA hybridization), unless required for appropriate description. DNA barcoding approach is gaining popularity for assessing microbial diversity. Though only limited datasets (especially for eukaryotic microbes) are currently available, the scenario is improving due to faster and cheaper sequencing methods.

\section{Marine microbes under the three domains of life}

\section{Bacteria}

Bacteria (Domain Bacteria) appear to have branched out very early on the tree of life and are genetically distinct from archaea (Domain Archaea) and eukaryotes (Domain Eukarya). Bacteria are abundant in all parts of the ocean and are vital to life on earth because they ensure the recycling of essential nutrients in oceanic food webs. Although bacteria have such a key role in sustaining basic functions in the marine environment, very little is known about their biology since only a small fraction (average 1\%) can be cultured under laboratory conditions. This is even more evident when considering that $>80 \%$ of all bacterial isolates from marine environment belong to four bacterial phyla: the Proteobacteria, Firmicutes, Actinobacteria, and Bacteroidetes.

Bacteria constitute a major part of the organic matter that feeds countless bottom-dwelling animals. Organic particles sinking in the water column are composed mostly of bacteria. Bacteria feed primarily on dead organic material. Some bacteria, however, are photosynthetic (cyanobacteria). Because of their size, cyanobacteria are believed to be the most abundant photosynthetic organisms in the ocean. In addition to being free-living, some bacteria have evolved to live in close association with other marine organisms. Many of the organelles found in eukaryotic organisms evolved from symbiotic bacteria. Examples of symbiotic bacteria include those involved in the digestion of wood by shipworms, those responsible for bioluminescence and those found in association with mussels, clams and tubeworms that live around hydrothermal vents. Most organic matter in oceans is decomposed by bacteria.

\section{Nutritional types}

- Photosynthetic Cyanobacteria (blue-green bacteria): Photosynthetic bacteria which are found in environments high in dissolved oxygen, and produce free oxygen, store excess photosynthetic products as cyanophycean starch and oils. primary photosynthetic pigments in cyanobacteria are chlorophyll a and chlorophyll b , accessory pigments include carotenoids and phycobilins.

- Other photosynthetic bacteria : Anaerobic green and purple sulfur and non-sulfur bacteria do not produce oxygen and the primary photosynthetic pigments are bacteriochlorophylls. Sulfur bacteria are obligate anaerobes (tolerating no oxygen) while non-sulfur bacteria are facultative anaerobes (respiring in low oxygen or in the dark and photosynthesizing anaerobically in the presence of light).

- Chemosynthetic bacteria : Use energy derived from chemical reactions that involve substances such as ammonium ion, sulfides and elemental sulfur, nitrites, hydrogen, and ferrous ion. Chemosynthesis is less efficient than photosynthesis, so rates of cell growth and division are slower. Found around hydrothermal vents and some shallower habitats where needed materials are available in abundance.

- $\quad$ Heterotrophic bacteria: Decomposers that obtain energy and materials from organic matter in their surroundings and return many chemicals to the marine environment through respiration and fermentation. Heterotrophic bacteria populate the surface of organic particles suspended in the water by secreting mucilage (glue-like substance)

\section{Nitrogen fixation and nitrification}

- Nitrogen fixation: Process that converts molecular nitrogen dissolved in seawater to ammonium ion, a major process that adds new usable nitrogen to the sea. Only some cyanobacteria and a few archaeons with nitrogenase (enzyme) are capable of fixing nitrogen.

- Nitrification: Process of bacterial conversion of ammonium $(\mathrm{NH} 4+)$ to nitrite (NO2) and nitrate (NO3-) ions. Bacterial nitrification converts ammonium into a form of nitrogen usable by other primary producers (autotrophs) 


\section{Archaea}

Archaea (Domain Archaea) are among the simplest, most primitive forms of life. Oldest fossils ever found (3.8 billion years old) appear similar to archaea. Archaea are prokaryotes, unicellular organisms that lack a nucleus and other membranebound organelles. These organisms are thought to have had an important role in the early evolution of life. Archaea were discovered first in extreme environments on land (hot sulfur springs, saline lakes, and highly acidic or alkaline environments) and were hence called as "Extremophiles". Archaea were subsequently found in extreme marine environments, such as in very deep water, where they survive at pressures of 300-800 atmospheres. Some archaea live at the high temperatures of hydrothermal vents, and cannot grow in temperatures under $70-80^{\circ} \mathrm{C}\left(158-176^{\circ} \mathrm{F}\right) ;$ One hydrothermal vent archaeum can live at $121^{\circ} \mathrm{C}\left(250^{\circ} \mathrm{F}\right)$, the highest of any known organism.

\section{Nutritional types}

Archaea includes photosynthesisers, chemosynthesisers and heterotrophs. most are methanogens, anaerobic organisms that metabolise organic matter for energy, producing methane as a waste product. Halobacteria (photosynthetic), thrive at high salinities, trap light using bacteriorhodopsins (purple proteins).

\section{Microbial Eukaryotes}

While all prokaryotes (domains Archaea and Bacteria) are unicellular, eukaryotes include both uni-cellular and multi-cellular organisms. Marie microbial eukayotes belong to Kingdoms Protista, Fungi, Plantae and Animalia. Most microbial marine eukaryotes belong to the Kingdom Protista - can be autotrophic or heterotrophic, unicellular or multicellular comprising algae, diatoms, dinoflagellates, zooxanthellae, coccolithophorids, foraminiferans, radiolarians, ciliates and marine fungi.

\section{Viruses}

Although they may not technically constitute a living organism, viruses are a critical component of the marine food web. Viruses are particles made up of nucleic acid (RNA or DNA) protected by a protein coat. They are parasites that reproduce and develop only with the aid of a living cell. Organisms are defined by their capacity of independent cell division and therefore possess the full machinery for the duplication of DNA and for the binary division of the cell. Viruses, in contrast, require a living organism in order to duplicate their genetic material and to synthesize the virus particle. They do not, therefore, belong to any of the three domains of life. Nevertheless, viruses are of prime importance for generating and maintaining diversity amongst living organisms in all three domains of life through the mediation of horizontal gene transfer. They are also critical for the functioning and balance of the microbial food web and biogeochemical cycles by facilitating the production of nutrients for growth and maintaining high diversity, all by preventing the dominance of the most successful microorganisms. They parasitize bacteria and plankton releasing organic matter into the ocean. Viruses may be responsible for half of the bacterial mortality in aquatic ecosystems and substantial amounts in phytoplankton. The amount of viruses in a given environment is directly related to the abundance of the microbial life, which they invade.

\section{Factors that impact marine microbial diversity}

Nearly every measurable physical, chemical, and biotic variable in the marine environment has been found to alter microbial diversity. The major variables affecting the marine microbial diversity include, turbulance, light, temperature, nutreints, salinity, pH, UV and solar influx, surfaces and interfaces, redox potencial, metals as well as presence of macroorganisms such as invertebates and macroalgae. For the most part, the extent to which each of these influences actually operates in the environment and the contexts in which they are important remain to be determined. Climate change, which will be felt by marine microbial communities as changes in ocean temperatures, will undoubtedly alter the diversity of communities in unforeseen ways. Climate change should be considered a major top-down controller of microbial communities. Pollution, including nitrogen inputs due to anthropogenic activities, also impact marine microbial diversity. Anthropogenic nitrogen inputs to the oceans now comprise about half the total nitrogen inputs to the oceans, a circumstance that has resulted in vast dead zones in coastal areas and an increased incidence of harmful algal blooms.

\section{Marine microbes and bioactive compounds}

The marine environment is emerging as a 'gold mine' for novel bioactive compounds. Marine derived natural products present an enormous range of novel chemical structures and provide an interesting and challenging blueprint for creating new entities via synthetic chemistry. Marine invertebrates and plants, in particular, represent an environment rich in microorganisms that produce compounds with bioactive properties including antibacterial, antifungal, antiviral, anticancer, antifouling and antibiofilm activities. However, only $1 \%$ of these microorganisms can be isolated using traditional culture techniques, which has been a major bottleneck when mining the marine environment for novel bioactive molecules. Marine microorganisms have immense potential for providing services and products for human society which is not exploited to any significant extent. There is a world of microorganisms in the marine environment to discover, understand and put to good use.

\section{Suggested reading}

Hughes, J. B., Hellmann, J. J., Ricketts, T. H. and Bohannan, J. M. 2001. Counting the uncountable: statistical approaches to estimating microbial diversity. Appl. Environ. Microbiol., 67: 4399-4406.

Martin, A. P. 2002. Phylogenetic approaches for describing and comparing the diversity of microbial communities. Appl. Environ. Microbiol., 68 :3673- 
3682.

Stach, J. E. M., Maldonado, L. A., Masson, D. G., Ward, A. C., Goodfellow, M. and Bull, A. T. 2003. Statistical approaches for estimating actinobacterial diversity in marine sediments. Appl. Environ. Microbiol., 69: 6189-6200.

Pedros-Alio, C. 2006 Marine microbial diversity: can it be determined? Trends in Microbiology, 14(6): 257-263.

Fierer, N. 2008. Microbial biogeography: patterns in microbial diversity across space and time. In: Zengler K. (Ed.). Accessing uncultivated microorganisms: from the environment to organisms and genomes and back. ASM Press, Washington DC, p. 95-115.

Koeppel, A. Perry, E.B., Sikorski, J Krizanc, D. Warner, A, Ward, D.M 2008. Identifying the fundamental units of bacterial diversity: a paradigm shift to incorporate ecology into bacterial systematics. Proceedings of National Academy of Sciences USA, 105(7): 2504-2509.

Zinger, L., Gobet, A. and Pommier, T. 2012. Two decades of describing the unseen majority of aquatic microbial diversity. Molecular Ecology, 21(8): 1878-1896. 